\title{
Erratum
}

\section{Microwave-Assisted Rhodium-Catalyzed Decarbonylation of Functionalized 3-Formyl-2H-chromenes: A Sequence for Functionalized Chromenes like Deoxycordiachromene}

Manuel C. Bröhmer, Nicole Volz, Stefan Bräse* Synlett 2009, 1383.

In the original Scheme 1 the Scheme title was incomplete. The correct title is the following:

Scheme 1 Strategies for the synthesis of chromenes based on $\alpha, \beta$-unsaturated aldehydes.

We apologize for this mistake. 